\title{
Philosophiques
}

\section{Émotions et les conditions de l'autonomie individuelle}

\section{Christine Straehle}

Volume 45, numéro 2, automne 2018

URI : https://id.erudit.org/iderudit/1055277ar

DOI : https://doi.org/10.7202/1055277ar

Aller au sommaire du numéro

Éditeur(s)

Société de philosophie du Québec

ISSN

0316-2923 (imprimé)

1492-1391 (numérique)

Découvrir la revue

Citer ce document

Straehle, C. (2018). Émotions et les conditions de l'autonomie individuelle.

Philosophiques, 45(2), 507-512. https://doi.org/10.7202/1055277ar d'utilisation que vous pouvez consulter en ligne.

https://apropos.erudit.org/fr/usagers/politique-dutilisation/ 


\title{
Émotions et les conditions de l'autonomie individuelle
}

\author{
CHRISTINE STRAEHLE
}

Université d'Ottawa

Christine.straehle@uottawa.ca

Le livre de Christine Tappolet, Emotions, Values, and Agency, offre un riche argumentaire pour une reconceptualisation des émotions. Plus spécifiquement, Tappolet soutient que les philosophes, mais aussi d'autres penseurs, doivent repenser le rôle des émotions dans les délibérations menant aux actions. Plutôt que d'emprunter les thèses traditionnelles qui se présentent de plus en plus en philosophie et dans les disciplines cognitives sous le titre de théorie des affects, Tappolet avance de façon convaincante que les émotions donnent des raisons justificatives de l'action. Ici, je me concentrerai sur l'argument de Tappolet selon lequel les émotions sont une source potentielle d'action autonome. Les liens entre émotions, autonomie individuelle et agentivité sont discutés plus précisément au chapitre $\mathrm{V}$ du livre.

Ainsi, ce chapitre propose trois thèses principales: «Les émotions peuvent être considérées, quand tout se déroule bien, comme des perceptions de raisons pratiques. [...] Les émotions confèrent une justification épistémique prima facie aux croyances évaluatives [...] Les émotions permettent non seulement de détecter des raisons, mais également, lorsque certaines conditions sont en place, de permettre la réceptivité aux raisons (ce qu'on appelle la reason-responsiveness) ${ }^{1}$.»

Je pense que la thèse plus générale de ce chapitre n'est pas controversée: comme nous indique Tappolet, il suffit de penser à l'aide que nous sommes en mesure d'offrir à nos amis ou aux êtres chers pour voir que nos émotions à leur égard sont en lien avec nos raisons d'agir d'une façon plutôt que d'une autre. Cela n'implique point que nous suivions aveuglément nos émotions, mais que notre souci pour autrui nous offre des raisons à partir desquelles nous agissons.

\section{Émotions occurrentes}

Je suis toutefois plus sceptique à l'égard d'un autre argument que Tappolet avance dans sa discussion. En discutant et réfutant la conception rationaliste de l'agentivité, elle soutient que, plutôt que d'abandonner l'idée que les émotions peuvent être à la source d'actions basées sur des raisons, nous avons besoin "[d']habitudes pratiques et épistémiques bien ajustées, telles que la personne en question n'agirait pas sur la base de ses émotions si elle avait des raisons de croire que ces dernières l'induiraient en l'erreur ${ }^{2}{ }^{\star}$.

1. C. Tappolet (2018), Précis, infra p. 465.

2. C. Tappolet (2018), infra p. 465. 
Il est important de se rappeler l'intérêt que Tappolet porte aux émotions occurrentes, à la différence de celui qu'elle porte aux dispositions émotionnelles. De fait, la théorie perceptuelle de Tappolet considère les types d'émotions qui nous saisissent, telles la peur et la colère, lorsque nous sommes confrontés à des phénomènes redoutables ou irritants, plutôt que les types d'émotions qui nous maintiennent dans le temps, comme l'amour ou la sollicitude. D'ailleurs, une bonne partie du premier chapitre explique comment les émotions occurrentes sont sujettes à un examen critique.

Néanmoins, je me demande dans quelle mesure la fonction de détection des raisons, que Tappolet attribue aux émotions, opère lorsque l'on considère les émotions occurrentes. Évidemment, une grande part de la réponse à cette question dépend de la forme que prendront les "habitudes pratiques et épistémiques» dont la source se trouve dans les vertus agentielles que Tappolet pose au fondement de sa conception de l'agentivité; ainsi, j'ai hâte de voir les travaux futurs qui tâcheront d'étoffer la définition des habitudes pratiques et épistémiques dont il est question.

\section{Émotions, autonomie et confiance en soi}

Je me tournerai maintenant vers un problème que la théorie de la vertu agentielle soulève dans le contexte de l'autonomie individuelle et de l'agentivité.

Tappolet passe en revue certains écrits des auteurs les plus importants qui se sont penchés sur la question de l'autonomie individuelle. Joseph Raz nous propose une de mes thèses favorites pour illustrer ce que l'autonomie signifie quand il la décrit comme le fait «d'être partiellement l'auteur de [sa] vie »3 .

Dans la continuité de la thèse de Tappolet, je crois qu'il est juste de dire que plusieurs soutiennent le lien entre autonomie et auto-gouvernance et, de manière plus importante encore, auto-constitution ${ }^{4}$. En guise d'illustration, reprenons l'exemple d'une personne et de son ami. Plus tôt, je disais que la considération pour un ami, ou disons le soin qu'un parent porte à son enfant, fait partie intégrante de la façon dont ce dernier agira. Le souci pour autrui nous donne des principes par rapport auxquels nous décidons de la manière dont nous vivons notre vie.

En l'occurrence, je propose que nos actes et la façon dont nous y réfléchissons ne sont pas de simples mesures temporaires, mais, à l'évidence, représentent comment nous prévoyons mener notre vie. À cet égard, ils sont l'expression de notre autonomie.

Dans le même ordre d'idées que Raz, et en allant plus loin, je suggère que les émotions ne sont pas seulement une expression de notre autonomie, mais que notre attachement à un enfant ou à un ami nous aide également de

3. J. Raz (I986) The Morality of Freedom, Oxford, Clarendon Press, at p. 370. Nous traduisons.

4. À ce sujet, voir entre autres C. Korsgaard (2009): Self-Constitution - Agency, Identity, and Integrity, Oxford, Oxford University Press. 
manière importante à nous constituer. Autrement dit, l'attachement ou le souci éprouvé pour autrui nous donne des raisons qui nous aident à guider nos pensées et nos actions. C'est d'ailleurs l'argument que présente Tappolet, et nous sommes d'accord à ce sujet.

Rappelons alors que la thèse du réalisme perceptuel défendu par Tappolet nous commande d'évaluer nos émotions afin de déterminer si elles sont appropriées au type de situation auquel nous faisons face. Dans un scénario pareil, cependant, il importe de se demander jusqu'à quel point nos émotions servent encore la fonction de constitution du soi que nous leur conférons par ailleurs. Prenons un exemple: un père qui se soucie du bien-être de sa fille. Admettons que l'ensemble de son entourage lui fasse valoir qu'il développe le syndrome du parent hélicoptère: il plane littéralement audessus de toutes les relations d'amitié et des interactions que sa fille entretient, il intervient dans toutes ses décisions et il se met à mal pour s'assurer qu' «elle est en sécurité ». Son comportement cesse d'être adéquat si l'idée d'élever des enfants est de leur permettre de se développer en fonction de leur propre personne. Face à ce genre de critiques, comment cet héli-père devrait-il réagir?

Il y a au moins deux réponses possibles qui vont de pair avec la désapprobation que manifeste l'entourage de l'héli-père, et avec le mode veille qui doit être en place pour qu'on puisse parler de réceptivité à l'égard de nos raisons (une troisième réponse serait de disqualifier la désapprobation à l'égard du parent hélicoptère, mais il s'agirait alors de désavouer le processus épistémique). La première réponse consisterait pour l'héli-père à réviser sa façon d'incarner ses émotions - nous pouvons imaginer, de façon cohérente avec la thèse de l'adéquation, que le père en question s'aperçoit qu'il est excessif et modère son comportement. Une deuxième réaction, cependant, pourrait être que l'héli-père souffre d'une perte importante de confiance en soi.

Afin d'illustrer cette dernière réaction, mettons en scène une situation différente. Imaginez que, plutôt qu'être un père envahissant, vous êtes un patient hospitalisé pour le traitement d'une maladie sévère. Les médecins offrent le type d'informations censées vous aider à prendre une décision au sujet des procédures que vous désirez entreprendre. Votre conception de vous-même correspond à celle d'une personne raisonnable, qui n'est pas facilement en proie à des peurs irrationnelles ou à des réactions fortes et soudaines. En d'autres mots, vous pensez posséder et démontrer les types d'habitudes épistémiques et pratiques qui distinguent l'agent possédant des vertus agentielles.

Cependant, à la lumière des procédures proposées, vous vivez de la panique, accompagnée des manifestations physiques qui y sont associées: perte de sommeil, cauchemars, bouche sèche, manque d'appétit, etc. Comment devriez-vous réagir, ou - pour parler en termes plus philosophiques - comment devrait-on penser cette réaction émotionnelle? 
Je pense qu'un des effets collatéraux du modèle avancé par Tappolet pourrait être la perte d'un ingrédient important de ce qui constitue l'autonomie pour les agents individuels. Autrement dit, et toujours selon le modèle proposé, il se peut qu'un acteur perde confiance en soi lorsqu'il essaie d'évaluer ses émotions. Je considère la confiance en soi comme un aspect important et prédominant de l'agentivité et de l'autonomie individuelle. Cependant, je désire surtout signaler que si nous adhérions au processus d'autoévaluation de nos émotions et au protocole exigé pour qu'il puisse y avoir réceptivité aux raisons, nous pourrions nous mettre à douter de nousmêmes, et cela au point de nuire à notre capacité de prendre des décisions autonomes.

Tappolet anticipe les grandes lignes de cette objection: dans sa discussion au chapitre III, elle note qu'il ne revient pas à ceux qui défendent la thèse de la réceptivité aux raisons de dire aux autres comment agir. Toutefois, s'il est vrai que les émotions font partie de notre auto-constitution, il n'est pas évident qu'un manque de confiance en soi pourrait ne pas être problématique à cet égard. Autrement dit, la question n'est pas celle de recommander ou non aux gens comment agir, mais plutôt celle d'imposer ou non des conditions à la vie émotionnelle qui pourraient miner les fondements de l'autonomie. Si cela résultait de l'auto-évaluation de nos émotions, l'argument en faveur des émotions comme perceptions de raisons pratiques risquerait d'échouer.

\section{Connaissance de soi, vulnérabilité et émotions contingentes}

Concluons sur un dernier point. Ce dernier touche à la connaissance de soi qui serait accessible aux individus et qui forme une partie importante de tout témoignage d'authenticité. Selon Tappolet, un agent autonome est tel que "ce qui peut être considéré comme un soi authentique, et non pas quelque force étrangère ou bien externe (comme un mari abusif ou une socialisation dégradante) ou bien interne (comme un désir compulsif) dirige ses pensées et ses actions ${ }^{5}$ ».

Cela est certainement vrai. Néanmoins, il est frappant que la thèse de la vertu agentielle présuppose que nous acquérions la connaissance de soi. La connaissance de soi me semble faire partie intégrante du récit défendu par Tappolet - après tout, nous sommes appelés à nous auto-évaluer afin de savoir si nos émotions sont appropriées dans une situation spécifique. Ainsi, lorsque nous ressentons de la peur devant un chien qui s'est mis à notre poursuite, il s'agit d'évaluer dans quelle mesure le petit terrier est véritablement capable de nous blesser plutôt que simplement pincer notre jambe. Pour amorcer ce type d'évaluation, nous devrions savoir et reconnaitre un certain nombre de faits à propos de nous-mêmes. À titre d'exemple,

5. C. Tappolet (2016) Emotions, Values, and Agency, Oxford, Oxford University Press UK, I90. Nous traduisons. 
il peut être important de nous rappeler que nous avons été mordus par un chien semblable étant enfant, ou que notre professeur détesté avait un comparable, etc. - soit des faits sur nous-mêmes qui auraient tendance à influencer nos réactions. Nous pourrions même nous rendre compte que notre réaction n'est basée sur rien.

Autrement dit, il nous faut avoir accès à ce savoir introspectif afin de pouvoir accéder au genre de raisons que nous pourrions avoir pour certaines émotions. Sans cet accès, il est fort peu probable de s'apercevoir que nous suivons une "force interne", telle la compulsion, plutôt que notre soi authentique.

Imaginons maintenant une situation de "gaslighting ", ou détournement cognitif. Le détournement cognitif est une condition psychologique qui fut nommée ainsi à la suite du film de George Cukor, Gaslight, en I944. On y voit Charles Boyer dans le rôle d'un mari qui convainc sa femme, jouée par Ingrid Bergman, qu'elle hallucine et qu'elle imagine une série de choses comme des variations dans l'éclairage au gaz, qui sont pourtant bien réelles. Depuis lors, le terme "gasligthing" a fait son entrée dans la terminologie des manuels de psychologie, décrivant une forme de manipulation qui vise à semer le doute chez un individu ou un groupe ciblé dans le but de lui faire questionner ses souvenirs, ses perceptions et sa santé mentale. Ce phénomène a aussi été discuté par les philosophes qui s'intéressent à l'autonomie individuelle ${ }^{6}$.

La question que je souhaiterais poser concerne la façon dont nous pouvons savoir que nos émotions sont des émotions authentiques, plutôt que des émotions que nous éprouvons sous l'effet de la manipulation. Il me semble que la théorie perceptuelle des émotions de Tappolet nous offre deux options pour répondre à l'inquiétude concernant cette vulnérabilité individuelle dans le domaine émotionnel. Une option serait de penser la théorie de Tappolet comme une théorie objective de la façon dont les émotions peuvent servir l'agentivité. Toutefois, cela irait à l'encontre de certains passages de sa discussion sur les propositions par d'autres, notamment Kolodny, suggérant qu'il existe des raisons subjectives et des raisons objectives. Selon Tappolet, une telle différentiation est caduque.

La deuxième option serait de défendre que son modèle de lien entre raisons, émotions et autonomie comme un modèle normatif. Cependant, cela sonne faux lorsqu'on lit que "pour être réellement réceptifs aux raisons, nous devrions apprendre à écouter nos émotions ${ }^{7} »$. En fait, la proposition du livre, dans la mesure où je la comprends, suggère justement que le poids normatif repose sur la façon d'être réceptif aux raisons, et non pas sur la façon dont nous écoutons nos émotions.

6. Voir K. Abramson (20I4) «Turn in on the Light on Gaslighting», Philosophical Perspectives.

7. C. Tappolet (2016) I95. Nous traduisons. 
Voilà que quelques commentaires pour encourager Christine Tappolet à partager davantage ses idées sur le lien entre nos émotions et le soi, un sujet important en philosophie morale en terme général, et plus particulièrement en psychologie morale. 